\title{
Etanercept in psoriasis: the evidence of its therapeutic impact
}

\author{
Andrew Thomson
}

Core Medical Publishing, Knutsford, UK

\begin{abstract}
Introduction: Psoriasis is a chronic inflammatory skin condition for which there is no cure. Treatment options are designed to control the disease symptoms and improve patients' quality of life, and physical and mental function. Established treatments can be effective but are also limited by tolerability, convenience, cosmetic, and economic issues. Etanercept, a fully human soluble tumor necrosis factor (TNF) receptor protein, is a recently approved systemic treatment for chronic moderate to severe plaque psoriasis.
\end{abstract}

Aim: To evaluate the evidence for the therapeutic value of etanercept in psoriasis.

Evidence review: There is clear evidence that etanercept $25 \mathrm{mg}$ or $50 \mathrm{mg}$ twice per week reduces physician-assessed severity of psoriasis and can lead to clearing when compared with placebo. There is substantial evidence that etanercept improves patients' quality of life as determined by both disease-specific and generic instruments. Emerging evidence includes improvements in symptoms associated with depression and fatigue. The tolerability of etanercept in patients with psoriasis appears to be similar to placebo. Initial indications from clinical trials suggest that there is no increased risk of infection or malignancy in etanercept-treated patients with psoriasis. The most common adverse events are reversible injection site reactions. Economic evidence is at present limited, although intermittent etanercept $25 \mathrm{mg}$ is considered cost effective in patients with severe disease unsuitable for systemic treatment.

Clinical value: Etanercept is an effective and efficient treatment for patients with moderate to severe psoriasis that may be suitable for intermittent use.

Key words: etanercept, evidence, psoriasis, TNF inhibitor, treatment

Core evidence clinical impact summary for etanercept in psoriasis

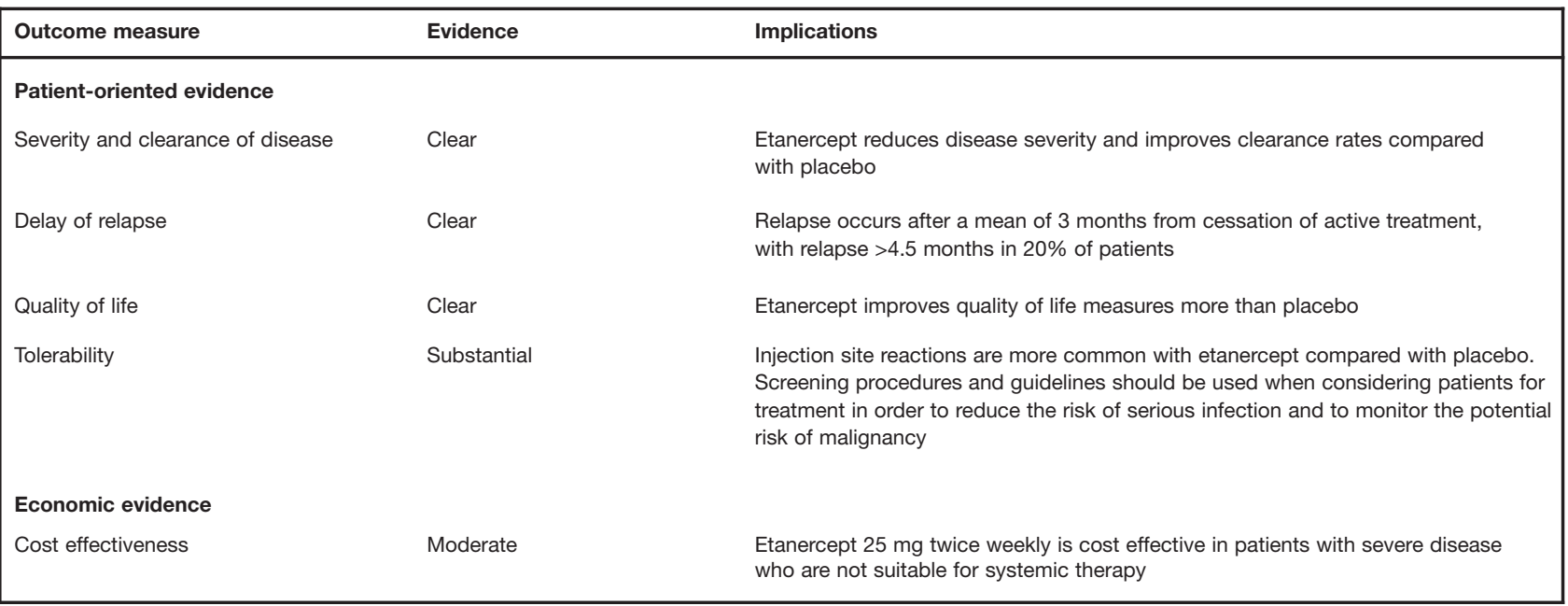




\section{Scope, aims, and objectives}

Psoriasis is a chronic condition of the skin affecting a significant proportion of the population that can lead to profound effects on quality of life and both physical and mental function. The condition can range in severity from the presence of a few localized lesions to more widespread disease affecting most of the body area, including the scalp, palms, soles of the feet, face, genitals, and nails. There is no cure for the disease, and the primary treatment goals are control of lesions and improvements in patients' quality of life.

Although mild forms of the disease may be managed effectively with existing topical therapies, established systemic or phototherapy may be required to treat moderate to severe disease. However, long-term treatment with traditional systemics and phototherapy may be associated with significant adverse events, not all patients are responsive, and many treatments are reimbursed at low rates. Therefore, there is a need for effective, well-tolerated, and conveniently administered treatments for the management of psoriasis. In 2004, etanercept (Enbrel ${ }^{\circledR}$; Amgen and Wyeth Pharmaceuticals) was the first tumor necrosis factor (TNF) receptor approved for the treatment of moderate to severe psoriasis. Although also approved for psoriatic arthritis (which occurs in approximately $40 \%$ of patients with extensive disease) the objective of this article is to review the clinical evidence base for the use of etanercept specifically for psoriasis.

\section{Methods}

The English language medical literature was searched in March 2006 in the following databases. The search strategy was "etanercept AND psoriasis" for articles published from January 1990 to November 2005 (inclusive). Nonhuman and in-vitro studies were excluded from the search.

- PubMed, http://www.ncbi.nlm.nih.gov/entrez/query.fgci

- EMBASE, http://www.datastarweb.com

- BIOSIS, http://www.datastarweb.com

- Database of Abstracts of Reviews of Effects (DARE), http://www.york.ac.uk/inst/crd/crddatabases.htm

- Cochrane Database of Systematic Reviews (CDSR), http://www.cochrane.org/index.htm

- Clinical Evidence (BMJ), http://www.clinicalevidence.com

- National Institute for Health and Clinical Excellence (NICE), http://www.nice.org.uk

- National Guideline Clearinghouse, http://www.guideline.gov

- Clinical trial register http://www.clinicaltrials.gov

A total of 35 publications (excluding guidelines) were identified from the search strategy (Table 1). Studies concerned exclusively
Table 1 | Evidence base included in the review

\begin{tabular}{|lcc|}
\hline Category & \multicolumn{2}{c|}{ Number of records } \\
\cline { 2 - 3 } & Full papers & Abstracts \\
\hline Initial search & 16 & 19 \\
records excluded & 5 & 7 \\
records included & 11 & 12 \\
Additional papers identified & 2 & 0 \\
Search update, new records & 111 & 0 \\
records excluded & 106 & 0 \\
records included & 5 & 0 \\
Level 1 clinical evidence & 1 & 5 \\
Level 2 clinical evidence & 7 & 4 \\
Level $\geq 3$ clinical evidence & 5 & 1 \\
trials other than RCT & 3 & 1 \\
case studies & 2 & 0 \\
Economic evidence & 0 & 12 \\
Total records included & 18 & 12 \\
\hline For definition of levels of evidence, see Editorial Information on inside back cover. \\
RCT, randomized controlled trial.
\end{tabular}

with palmoplantar pustular psoriasis, guttate psoriasis, or psoriatic arthritis were excluded. Following the search and manual checking of the references, 13 full papers and 12 meeting abstracts (from PubMed, BIOSIS, and EMBASE) were included in the evidence base. The search was repeated on September 22, 2006, yielding another five papers, and two more studies were identified, resulting in a total of 30 records in the evidence base.

\section{Disease overview}

Psoriasis is a chronic inflammatory skin condition that affects about $2 \%$ of the population (Pardasani et al. 2000). It is a disease that interferes with many normal daily activities, including use of the hands, walking, sleeping, and sexual activity. In fact, psoriasis has been reported to cause greater physical and mental distress compared with many other major diseases, including arthritis, chronic lung disease, chronic heart failure, and diabetes (Rapp et al. 1999). As such, it has a profound emotional and social as well as physical impact on quality of life (Krueger et al. 2001).

There are several types of psoriasis with plaque-type being the most common form, accounting for over $80 \%$ of cases. Guttate psoriasis occurs in about $10 \%$ of patients, and erythodermic and pustular types each occur in fewer than $3 \%$ of patients (Lebwohl 2003). Plaque psoriasis is characterized by sharply demarcated, erythematous, scaling plaques, typically affecting the elbows, knees, scalp, and intergluteal cleft. In some patients, lesions may appear on the palms and soles before other areas are affected, and lesions may occasionally appear on the genitals, face, and nails (Lebwohl 2003). 
Table 2 | Established treatment options for psoriasis

\begin{tabular}{|c|c|c|}
\hline Classification & Treatment & Risks and comments \\
\hline \multirow[t]{5}{*}{ Localized or topical treatments } & Topical corticosteroids, various potencies & Skin atrophy; rebound may occur on withdrawal \\
\hline & Vitamin D analogs & Skin atrophy \\
\hline & Coal tars & Strong smell and staining; may cause folliculitis \\
\hline & Anthralin & Staining; irritation to normal tissue \\
\hline & Tazarotene & Increased phototsensitivity; avoid use in women of childbearing potential \\
\hline \multirow[t]{5}{*}{ Generalized or systemic treatments } & UVB phototherapy & Acute phototoxicity \\
\hline & PUVA & Acute phototoxicity and cutaneous malignancy \\
\hline & Retinoids (acitretin) & Teratogenic; avoid use in women of childbearing potential \\
\hline & Methotrexate & Hepatotoxicity; acute neutropenia and pancytopenia \\
\hline & Cyclosporine & Renal toxicity \\
\hline
\end{tabular}

Psoriasis leads to considerable economic burden by affecting direct cost of care and also indirect cost of reduced productivity in both work and home environments. Visits to US physicians made by patients principally for care of psoriasis average 1.5 million visits per year (Stern 1996). A recent estimate of the direct costs of care for psoriasis (including psoriatic arthritis) in the USA determined the total cost to be \$US649.6 million for about 1.4 million individuals with clinically significant disease (\$US464 per patient) (Javitz et al. 2002). This estimate is likely to increase given the higher acquisition costs of newer biologic therapies that are now available for the treatment of psoriasis.

Recent investigations into the pathogenesis of psoriasis have led to the recognition of the importance that the immune system plays in the disease. This in turn has led to the development of biologic therapies that target cells and mediators that are implicated in inflammatory aspects of the disease.

\section{Current therapy options}

As there is no definitive cure for psoriasis, the goals of treatment include long-term improvement in the physical signs and secondary functional and psychologic effects, reduction in inflammation, and control of skin shedding. Improvement in patients' quality of life is also important given the serious impact the disease has on physical, mental, emotional, and social functioning (Krueger et al. 2001).

There are a number of established therapeutic options available which range from topical treatments for mild disease to systemic or phototherapy for more severe disease (Lebwohl 2003). The most commonly prescribed treatments for psoriasis in the USA and many other parts of the world are topical corticosteroids which are available in a number of strengths and forms (e.g. creams, sprays, gels). Other topical treatments include coal tar preparations, vitamin D analogs, and tazarotene (a retinoid and the most recent development in topical therapy) (Table 2).
Phototherapy or systemic therapy is used in patients whose psoriasis is not controlled adequately with topical treatments or whose disease is too widespread. Phototherapy options include ultraviolet $B$ (UVB) light or the combination of oral psoralen followed by irradiaton with UVA light (PUVA). Established systemic treatments include methotrexate, cyclosporin, and acitretin. The beneficial effects achieved with these drugs have led to the introduction of more selective agents following detailed investigations into the pathogenesis of psoriasis. Thus, targeted therapies that interfere with immunologic steps in the pathogenesis of the disease, such as inhibitors of T-cell activation and inhibitors of specific cytokines, have been developed. One such agent is etanercept, which blocks the action of TNF, an inflammatory cytokine that plays an important role in psoriasis (Bonifati \& Ameglio 1999).

Outcomes to determine effectiveness in psoriasis include both clinical and patient-reported measures. The Psoriasis Area and Severity Index (PASI) is an outcome measure commonly used by physicians in clinical studies (Ashcroft et al. 1999). It is a composite index indicating the severity of the three main characteristics of psoriatic plaques (erythema, scaling, and thickness, scored 0-4 for each), weighted by the amount of coverage (on a scale of 0-6) on the four main body areas (head, arms, legs, and trunk). The index ranges from 0 (no psoriasis) to 72 (most severe disease). Improvements in PASI of 50,75 , and $90 \%$ (PASI 50, 75, and 90, respectively) are often used as clinical outcomes.

Although PASI may be a popular measure in clinical studies, it is not often applicable to general dermatology practice. The Physician's Global Assessment (PGA) is more widely used in clinical practice (Jacob et al. 2005). This instrument corresponds to the physician's global assessment of changes in all psoriatic lesions compared to the baseline condition and is often helped by referral to photographs taken before treatment begins. It is common for the PGA to be reported on a scale of 0 to 5 ( 0 indicating no disease or clear of psoriasis, with higher 
scores indicating more severe disease). Worsening of disease can be denoted by a value of -1 (Shikiar et al. 2003). Both the PASI and PGA are highly correlated $(r>0.8)$ and have high overall reliability ( $>0.9)$ (Langley \& Ellis 2004).

Because of the marked negative influence that psoriasis can have on patients' quality of life, it is important to determine patient-reported outcomes. Examples of these measures include disease-specific measures, such as the Psoriasis Symptom Assessment (PSA) scale, Patient's Global Assessment of Psoriasis, the Dermatology Life Quality Index (DLQI), and generic instruments such as the Short Form-36 Health Survey (SF-36).

The DLQI contains ten items relating to the patient's skin with a score range of 0 to 30 (higher scores indicating poor quality of life) and can be self-administered. The instrument contains six subscales: daily activities, symptoms and feelings, work/school, leisure, personal relationships, and treatment satisfaction (Shikiar et al. 2003).

In order to determine disease severity, it is necessary to assess the sum of the effect of the signs and symptoms of psoriasis and the impact that it has on the patient's life. It is possible to define severe disease as a PASI score of $>10$ (or an involvement of $>10 \%$ of the body surface area involved) and a DLQI score of $>10$ (Finlay 2005).

\section{Unmet needs}

Despite the availability of many treatments for psoriasis, their adverse effects or inadequate efficacy have created the need for more effective, and better tolerated therapies (Lebwohl 2003). For example, in a survey of 6194 patients with severe psoriasis conducted in 1998, 40\% felt frustrated with the ineffectiveness of their current treatment and $32 \%$ reported that treatment was not aggressive enough (Krueger et al. 2001). A more recent survey of 4.5 million adults in the USA indicated that over $22 \%$ of patients with psoriasis had substantial dissatisfaction with their treatment (Stern et al. 2004). This may be due in part to serious risks or other factors being associated with a number of established treatments (Table 2).

Topical treatments require lengthy (at least overnight) skin exposure and can be inconvenient and awkward to apply, especially if a large PSA is affected. Tars are messy and stain the skin, and few patients can tolerate the smell. Attempts to produce effective nonstaining preparations that are not greasy have been unsuccessful. Lengthy use of topical corticosteroids can also lead to skin atrophy, systemic toxicity, and tachyphylaxis or flare-up in disease after discontinuation. PUVA is a very effective treatment but is associated with the risk of developing cutaneous malignancy. Several highly effective systemic treatments are available, but they are also associated with a number of adverse side effects. For example, acute neutropenia and chronic hepatotoxicity can occur with methotrexate, and long-term use of cyclosporin is associated with nephrotoxicity (Pardasani et al. 2000; Lebwohl 2003).

Therefore, there is still the need for effective, well-tolerated, and convenient options for treating psoriasis. One type of treatment that may address these requirements is biologic targeted therapy. Etanercept is a recombinant human TNF-receptor fusion protein which inhibits the interaction of endogenous TNF with cell-surface receptors. It was first approved for the treatment of psoriatic arthritis by the US Food and Drug Administration (FDA) in January 2002. This was followed in May 2004 with approval from the FDA for the treatment of moderate to severe plaque psoriasis in patients who are candidates for systemic therapy or phototherapy. In September 2004, approval was gained in the European Union for etanercept to be used for the treatment of adult patients with moderate to severe plaque psoriasis who failed to respond to, have a contraindication to, or are intolerant of other systemic therapies.

\section{Clinical evidence with etanercept in psoriasis}

\section{Clinical outcomes}

There is clear evidence that treatment with etanercept can achieve rapid, significant improvements in the activity and severity of psoriasis as assessed by physicians.

\section{Improvement in PASI}

Encouraging results in PASI obtained in a phase II trial with etanercept in 60 patients with psoriatic arthritis have been confirmed in a meta analysis which evaluated data from one phase II (Gottlieb et al. 2003) and two phase III randomized controlled studies (Leonardi et al. 2003; Papp et al. 2005) with etanercept specifically in patients with psoriasis. However, this analysis has only been published in abstract form (Gordon et al. 2004; van der Kerkhof et al. 2004a), making full evaluation difficult. The inclusion criteria for the studies were almost identical: patients $(n=1187)$ with stable chronic plaque psoriasis involving at least $10 \%$ body surface area who had received (or were candidates for) photo- or systemic therapy. In this analysis, a significantly higher proportion of etanercept-treated patients (33 and $49 \%$ treated with $25 \mathrm{mg}$ and $50 \mathrm{mg}$ twice weekly, respectively) achieved the primary endpoint of $\geq 75 \%$ improvement in PASI at 12 weeks compared with placebo $(P<0.0001)$. In addition, a higher proportion of patients achieved clearing of disease ( $\geq 90 \%$ improvement in PASI) after 12 weeks with etanercept $50 \mathrm{mg}$ twice weekly compared with placebo (21 vs 1\%). The effects of etanercept on improvements in PASI are also seen in each of the constituent studies of this analysis (Table 3).

Results from a further randomized controlled trial of etanercept $50 \mathrm{mg}$ twice weekly has also confirmed that etanercept improves PASI scores (Tyring et al 2006). PASI 75 was achieved in $47 \%$ of etanercept recipients compared with $5 \%$ of those receiving placebo, and PASI 90 by $21 \%$ and $1 \%$, respectively $(P<0.0001$ for both endpoints). A once-weekly dose of etanercept $100 \mathrm{mg}$ has been shown to be as effective as $50 \mathrm{mg}$ twice weekly, with PASI 50 achieved in $78 \%$ of 51 and $74 \%$ of 50 patients, respectively, after 12 weeks (Cassano et al. 2006).

Etanercept efficacy was also maintained after reducing the dose in an open-label extension of a phase III study (Papp et al. 2005). 
Table 3 | Effect of etanercept on Psoriasis Area and Severity Index (PASI) score in patients with psoriasis

\begin{tabular}{|c|c|c|c|c|}
\hline Level of evidence & Reference & Design & Treatment (mg) & Patients achieving PASI $\geq 75$ at 12 weeks (\%) \\
\hline \multirow[t]{3}{*}{$1^{\mathrm{a}}$} & \multirow{3}{*}{$\begin{array}{l}\text { Gordon et al. 2004; } \\
\text { van der Kerkhof et al. } \\
2004 a\end{array}$} & \multirow{3}{*}{$\begin{array}{l}\mathrm{R}, \mathrm{DB}, \mathrm{PC}, 12 \text {-week study involving } \\
1187 \text { patients }\end{array}$} & ETN 25 biw & 33 \\
\hline & & & ETN 50 mg biw & 49 \\
\hline & & & PLA & $3(P<0.0001$ vs ETN $)$ \\
\hline $1^{a}$ & Strober et al. 2004 & $\begin{array}{l}\mathrm{R}, \mathrm{DB}, \mathrm{PC}, 12 \text {-week study involving } \\
415 \text { patients } \pm \text { previous systemic or } \\
\text { phototherapy }\end{array}$ & ETN 25 biw & $\begin{array}{l}33 \text { vs } 34 \text { (NS) for patients } \pm \text { previous systemic } \\
\text { or phototherapy, respectively }\end{array}$ \\
\hline \multirow[t]{2}{*}{2} & \multirow[t]{2}{*}{ Mease et al. 2000} & \multirow{2}{*}{$\begin{array}{l}\mathrm{R}, \mathrm{DB}, \mathrm{PC}, 12 \text {-week study involving } \\
38 \text { patients (psoriasis } \geq 3 \% \text { BSA involvement) }\end{array}$} & ETN 25 biw & 26 \\
\hline & & & PLA & $0(P=0.0154$ vs ETN $)$ \\
\hline \multirow[t]{2}{*}{2} & \multirow[t]{2}{*}{ Gottlieb et al. 2003} & \multirow{2}{*}{$\begin{array}{l}\mathrm{R}, \mathrm{DB}, \mathrm{MC}, \mathrm{PC} 24 \text {-week study involving } \\
112 \text { patients }\end{array}$} & ETN 25 biw & 30 \\
\hline & & & PLA & $2(P<0.0001$ vs ETN $)$ \\
\hline \multirow[t]{4}{*}{2} & \multirow[t]{4}{*}{ Leonardi et al. 2003} & \multirow{4}{*}{$\begin{array}{l}\mathrm{R}, \mathrm{DB}, \mathrm{PC}, 24 \text {-week study involving } \\
672 \text { patients }\end{array}$} & ETN 25 qw & 14 \\
\hline & & & ETN 25 biw & 34 \\
\hline & & & ETN 50 biw & 49 \\
\hline & & & PLA & $4(P<0.0001$ vs ETN $)$ \\
\hline \multirow[t]{3}{*}{2} & \multirow[t]{3}{*}{ Papp et al. 2005} & \multirow{3}{*}{$\begin{array}{l}\text { R, DB, PC, 24-week (OL phase during } \\
\text { weeks 13-24) study involving } 583 \text { patients }\end{array}$} & ETN 25 biw & 34 \\
\hline & & & ETN 50 biw & 49 \\
\hline & & & PLA & $3(P<0.0001$ vs ETN $)$ \\
\hline \multirow[t]{2}{*}{2} & \multirow[t]{2}{*}{ Tyring et al. 2006} & \multirow{2}{*}{$\begin{array}{l}\mathrm{R}, \mathrm{DB}, \mathrm{MC}, \mathrm{PC}, 12 \text {-week study involving } \\
618 \text { patients followed by an } 84 \text {-week, } \\
\mathrm{OL} \text {, active treatment period }\end{array}$} & ETN 50 biw & 47 \\
\hline & & & PLA & $5(P<0.0001$ vs ETN $)$ \\
\hline \multirow[t]{2}{*}{2} & \multirow[t]{2}{*}{ Cassano et al. 2006} & \multirow{2}{*}{$\begin{array}{l}\mathrm{R}, \mathrm{DB}, 12-\text { week study involving } \\
101 \text { patients }\end{array}$} & ETN 50 biw & 54 \\
\hline & & & ETN 100 qw & 50 \\
\hline
\end{tabular}

After 12 weeks of double-blind treatment with etanercept $50 \mathrm{mg}$ twice weekly, patients $(n=179)$ were switched to $25 \mathrm{mg}$ twice weekly, and PASI 75 response at weeks 12 and 24 were compared. Most $(70 / 91 ; 77 \%)$ of the PASI 75 responders at week 12 maintained their response at week 24. Of those 21 patients who did not maintain PASI 75 by week 24 , three failed to maintain a PASI 50 response. At week 12, 88 patients had not achieved a PASI 75 response, however, by week $24,32 \%$ of the 88 patients had done so despite the decrease in etanercept dose. A similar pattern emerged in patients initially not achieving PASI 50 in an open-label study (Kreuger et al. 2006). Forty-three percent of 157 patients failing to achieve PASI 50 after 24 weeks of treatment with etanercept $50 \mathrm{mg} /$ week subsequently did so after 36 weeks, increasing to $55 \%$ after 60 weeks.

\section{Onset of action}

There is evidence that etanercept achieves efficacy within 2 to 4 weeks of starting treatment (Leonardi et al. 2003). Differences in the numbers of patients considered clear/almost clear of psoriasis and mean improvements in PASI with etanercept compared with placebo were evident within this time. PASI scores were improved significantly after 4 weeks of high-dose $(50 \mathrm{mg}$ twice weekly) and 8 weeks of medium-dose ( $25 \mathrm{mg}$ twice weekly) treatment compared with placebo. The proportion of patients assessed as being clear or almost clear of psoriasis was significantly different for all the etanercept groups by week 4 compared with the placebo group.

\section{Relapse}

In the study by Leonardi et al. (2003), patients classified as responders ( $\geq$ PASI 50 from baseline) were discontinued from treatment after 24 weeks and followed until their disease relapsed (loss of $\geq 50 \%$ of PASI improvement) (Gordon et al. 2006). Of 409 patients entering the withdrawal and retreatment period, 347 relapsed and received at least one further dose of etanercept, and 297 completed 12 weeks of retreatment. The median time to relapse was 85 days, with $25 \%$ of the responders not relapsing until after 145 days (Gottlieb et al. 2004c; Leonardi et al. 2004). In a subset of 252 patients who achieved PASI 75 after 24 weeks, median time to loss of this response was 57 days, and loss of PASI 50 occurred in a median of 91 days (Gordon et al. 2006). During the withdrawal period only one patient returned to $>125 \%$ of baseline PASI score. No cases of conversion of psoriasis morphology were seen. After 12 weeks, the overall effect of etanercept retreatment (mean PASI score 6.4) was similar to the initial treatment effect (mean PASI score 5.8), and $83 \%$ of patients achieving PASI 50 during initial treatment achieved the same response after 12 weeks of retreatment. Although from only one cycle of discontinuation and retreatment, these results suggest that etanercept may be used effectively in an intermittent or rotational approach to treat psoriasis. 
Table 4 | Effect of etanercept on other disease-related outcomes in patients with psoriasis

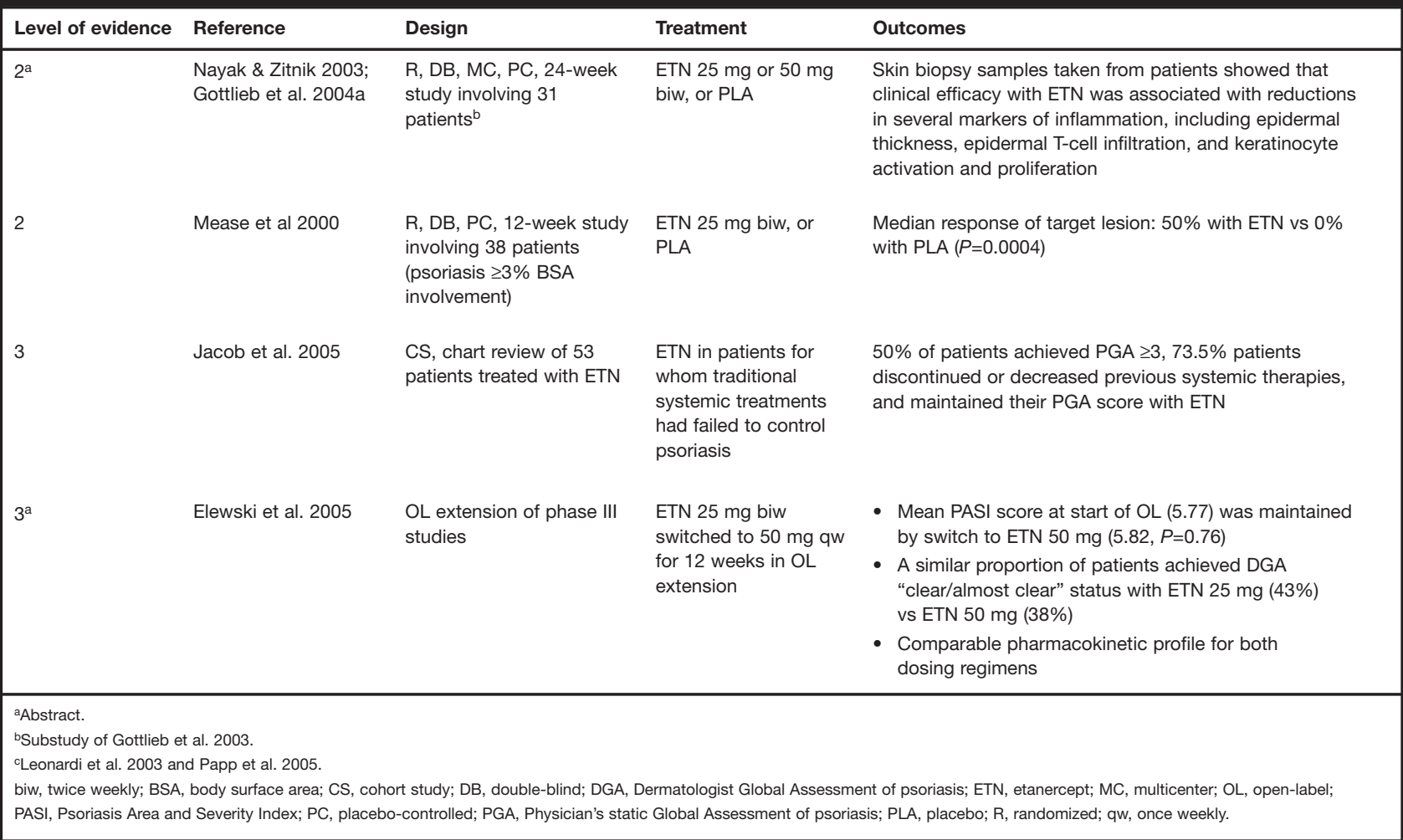

\section{Previously treated patients}

Results from the meta analysis showed that etanercept was as effective in patients with severe disease who had been treated previously with systemic or phototherapy as in those with no such history (Strober et al. 2004; Table 3). In 415 patients randomized to etanercept $25 \mathrm{mg}$ twice weekly, those with a history of systemic or phototherapy had significantly $(P>0.01)$ more severe disease (body surface area involvement 30 vs 21\%) of longer duration (21 vs 17 years), and higher baseline PASI score (19.2 vs 16.0), compared with those with no history $(P>0.01$ for all parameters). Despite these differences, the same proportions of patients in both groups achieved comparable PASI responses after 12 and 24 weeks of treatment (Table 3).

\section{Quality of life}

There is good evidence from randomized controlled trials that etanercept rapidly improves patient-reported disease and quality of life outcomes in psoriasis (Table 5).

A meta analysis of quality of life outcomes has been carried out following analysis of data from one phase II (Nayak \& Zitnik 2003; Gottlieb et al. 2004a) and two phase III randomized controlled studies (Leonardi et al. 2003; Papp et al. 2005) with etanercept. Results from this meta analysis have appeared as abstracts from meetings (Gordon et al. 2004; Singh et al. 2004; van der Kerkhof et al. 2004b).
The meta analysis showed that after 12 weeks of treatment the odds of achieving a clinically meaningful improvement in DLQI total score (defined as 5 points) were four to five times higher with either etanercept $25 \mathrm{mg}$ or $50 \mathrm{mg}$ twice weekly compared with placebo $(P<0.0001)$ (Singh et al. 2004). This is a reflection of the mean percentage improvement from baseline of 51.5 and $59.1 \%$ in DLQI scores with etanercept $25 \mathrm{mg}$ and $50 \mathrm{mg}$ twice weekly, respectively, compared with placebo treatment (Singh et al. 2004; van der Kerkhof 2004b). In addition, treatment with both doses of etanercept resulted in statistically significant improvements in each of the six subscales of the DLQI compared with placebo.

Improvements in DLQI were also achieved irrespective of whether patients had a history of previous treatment with systemic or phototherapy (Strober et al. 2004). As well as having more severe disease, patients with a history of previous systemic therapy also had a worse DLQI score at baseline compared with those who did not (11.9 vs $10.4, P<0.01)$. Yet after treatment with etanercept $25 \mathrm{mg}$ twice weekly, the improvements in DLQI after 12 (60 vs $54 \%$ ) and 24 weeks (64 vs $61 \%$ ) were not significantly different in previously treated or untreated patients.

Details of significant etanercept-related improvements in both disease-specific (DLQI) and generic (SF-36) quality of life measures from one of the constituent studies of the meta analysis have been reported recently (Krueger et al. 2005; Table 5). In this study etanercept produced improvements in patient-reported outcomes as early as 2 weeks after starting therapy. Compared 
with placebo-treated patients, a significantly greater proportion of etanercept-treated patients reported clinically important improvements ( $\geq 5$ points, or a score of 0 ) in DLQI scores at week $12(P<0.0001)$. In all six DLQI subscales, improvements in patients treated with etanercept were significantly greater than in those treated with placebo. The two subscales showing the greatest levels of improvement with etanercept were the symptoms and feelings and daily activities subscales $(P<0.0001)$.

Significant improvements in patients' functional status were also seen with SF-36 scores after 12 weeks of etanercept treatment. Both the physical and mental component scores were significantly higher in patients treated with etanercept compared with placebo-treated patients $(P<0.01)$. In addition, all eight subscales were improved significantly with etanercept compared with placebo; the greatest mean changes occurred in the measures of bodily pain $(P<0.0001)$ and social function subscales $(P<0.001)$. During the open-label phase of the study, patients who had originally received etanercept $50 \mathrm{mg}$ twice weekly maintained the improvement in patient-reported outcomes when switched to etanercept $25 \mathrm{mg}$ twice weekly.

In addition to improvements in DLQI, evidence is emerging that etanercept treatment may improve symptoms of depression and fatigue in patients with psoriasis (Tyring et al. 2006). In this study, depression was assessed by both the Beck Depression Inventory (BDI; patient-administered questionnaire) and the Hamilton rating scale for depression (Ham-D; administered by trained healthcare professionals). Although depression is reported to be a common finding in patients with psoriasis, it is notable in this study involving 618 patients that at baseline only a few patients in the etanercept and placebo groups had moderate to severe depression; 15 and $16 \%$, respectively, as determined by the BDI and $2 \%$ in each group as determined by the Ham-D. This may be due, in part, to strict study inclusion criteria discouraging the most depressed participants from enrollment (Tyring et al. 2006). At baseline, most patients had no (74\%, Ham-D score) or minimal (66\%, BDI scale) depression. Nevertheless, results from the study showed that more patients treated with etanercept achieved at least a $50 \%$ improvement in both of these scales after 12 weeks compared with placebo. Significant improvement in the BDI total score was achieved after 4 weeks of treatment with etanercept compared with placebo $(P=0.0062)$.

Patients with psoriasis in this study were also more fatigued compared with historic data for the general population (mean baseline functional assessment of chronic illness therapyfatigue [FACIT-F] score $3.78 \pm 11.2$ vs $43.6 \pm 9.4$ for the general population). After only 2 weeks of treatment with etanercept, patients showed a significantly greater improvement in FACIT-F score compared with placebo-treated patients $(P=0.0048)$. After 12 weeks of treatment, the mean improvement in score of 5.0 points for etanercept-treated patients was significant and clinically meaningful compared with placebo (1.9 points, $P<0.0001)$. Therefore, given the limitations of the baseline characteristics of the study population, the data suggest that there is limited evidence that etanercept treatment improves symptoms of depression and fatigue in patients with psoriasis.

\section{Other outcomes}

Statistically significant improvements in patient global, physician global, and target lesion assessments were reported to have been achieved with etanercept although results from these trials were available as abstracts only, and specific data were missing (Nayak \& Zitnik 2003; Gottlieb et al. 2004a). However, results from skin biopsy samples taken from a subset of patients (17 treated with etanercept, 14 treated with placebo) showed that markers of inflammation and other endpoints were reduced with etanercept compared with placebo treatment. For example, the mean reduction in epidermal thickness after 12 weeks of treatment was greater with etanercept (44\%) compared with placebo (7\%, $P<0.05)$. (Gottlieb et al. 2004a). There were also reductions in keratinocyte adhesion protein expression (keratinocyte ICAM-1), and epidermal T-cell (CD3) infiltration and proliferation (epidermal Ki 67) associated with etanercept treatment (Table 4).

Improvement in psoriatic skin lesions with etanercept was also seen in the study by Mease et al. (2000). The median response of a prospectively defined target lesion in the etanercept group was $50 \%$ compared with $0 \%$ in the placebo group $(P=0.0004)$ (Table 4). In addition, a chart review of 53 patients showed that treatment with etanercept also led to discontinuation or dose reduction in previous systemic therapies in nearly three-quarters of patients while maintaining their PGA score (Jacob et al. 2005).

\section{Tolerability and safety}

Reports of the tolerability of etanercept in patients with psoriasis have been limited to results from clinical trials, and long-term surveillance data have yet to appear in this population. In clinical studies involving patients with psoriasis, the incidence of adverse effects and infections with etanercept have generally been similar to placebo (Table 6).

Because of the mode of action, concerns have been raised regarding the potential for immunosuppression with TNF inhibitors (including etanercept), leading to infection (notably tuberculosis), malignancy, and other serious potential toxicities (Hochberg et al. 2005; Smith et al. 2005). Most of the data on the safety of etanercept have been generated from clinical studies and postmarketing surveillance in patients with rheumatoid arthritis, and in March 2003 the FDA Arthritis Advisory Committee (AAC) convened for an update on the safety of TNF inhibitors, including etanercept (ACR 2003a,b). The meeting focused specifically on the issue of neoplasia and lymphoma in patients with rheumatoid arthritis. As of the fourth quarter of 2002, 150000 patients had been treated with etanercept, providing 230000 patient-years of exposure and 70 reports of lymphoma. This incidence of lymphoma of 0.03 cases per 100 patient-years of exposure is equal to the normal population rate (ACR 2003a). However, patients with severe psoriasis have an increased risk (approaching that for patients with organ transplants) for lymphoproliferative disorders and should, therefore, be followed for any signs of malignancy irrespective of therapy.

Only $10 \%$ of cases associated with etanercept use, but nearly half $(48 \%)$ of all reported cases of tuberculosis, were reported from 
Table 5 | Effect of etanercept on patient-reported outcomes in patients with psoriasis

\begin{tabular}{|c|c|c|c|c|}
\hline Level of evidence & Reference & Design & Treatment & Outcomes \\
\hline \multirow[t]{2}{*}{$1^{\mathrm{a}}$} & $\begin{array}{l}\text { Singh et al. 2004; } \\
\text { van der Kerkhof et al. } \\
2004 b\end{array}$ & $\begin{array}{l}\mathrm{R}, \mathrm{DB}, \mathrm{PC}, 12 \text {-week study } \\
\text { involving } 1187 \text { patients }\end{array}$ & $\begin{array}{l}\text { ETN } 25 \text { mg, } 50 \text { mg biw, } \\
\text { or PLA }\end{array}$ & $\begin{array}{l}\text { OR for achieving a clinically meaningful improvement on DLQI: } \\
\text { - } 4.20(95 \% \mathrm{Cl} 3.14,5.63) \text { and } 5.87(95 \% \mathrm{Cl} 4.25,8.10) \text { for } \\
\text { ETN } 25 \mathrm{mg} \text { and } 50 \mathrm{mg} \text {, respectively, vs PLA }(P<0.0001)\end{array}$ \\
\hline & & & & $\begin{array}{l}\text { Mean improvement in DLQI at } 12 \text { weeks: } \\
\text { - } 51 \%(95 \% \mathrm{Cl} 44.6,58.1) \text { and } 59.1 \%(95 \% \mathrm{Cl} 51.6,66.6) \text { for } \\
\text { ETN } 25 \mathrm{mg} \text { and } 50 \mathrm{mg} \text {, respectively, vs PLA }(P<0.0001)\end{array}$ \\
\hline
\end{tabular}

Strober et al. 2004 involving 415 patients \pm previous systemic or phototherapy ${ }^{b}$

Leonardi et al. 2003

R, DB, PC, 24-week study involving 672 patients

Krueger et al. 2005

R, DB, PC, 24-week study (OL phase during weeks 13-24) involving 583 patients
ETN 25 mg qw (low dose), $25 \mathrm{mg}$ biw (medium dose), $50 \mathrm{mg}$ biw (high dose), or PLA for 12 weeks

ETN $25 \mathrm{mg}$ or $50 \mathrm{mg}$ biw, or PLA for 12 weeks All patients received ETN 25 mg biw during OL phase
R, DB, MC, PC, 12-week study involving 618 patients followed by an 84-week OL active treatment period
Cassano et al. 2006

Elewski et al. 2005
R, DB, 12-week study involving 101 patients

12-week OL extension of ETN phase III studies involving 265 patients
ETN 50 mg biw, or PLA

ETN 50 biw

ETN 100 qw

ETN $25 \mathrm{mg}$ biw switched to $50 \mathrm{mg}$ qw for 12 weeks in OL extension

Percentage improvement in DLQI:

- 60 vs $54 \%$ (NS) for patients and without previous systemic or phototherapy at 12 weeks

- 64 vs $61 \%$ (NS) for patients and without previous systemic or phototherapy at 24 weeks

Percentage improvement in DLQI:

- 47.2, 50.8, and 61.0\% in low-, medium-, and high-dose groups vs $10.9 \%$ for PLA $(P<0.0001)$ at 12 weeks

- 54.0, 59.4, and 73.8\% in low-, medium-, and high-dose groups

PtGA scores improved significantly in all ETN groups vs PLA $(P<0.001)$

DLQI at 12 weeks:

- Total score improved by $65-70 \%$ for ETN vs $6 \%$ for PLA $(P<0.0001)$

- Clinically meaningful improvements in DLQI achieved by $72-77 \%$ for ETN vs $26 \%$ for PLA $(P<0.0001)$

- All 6 subscales were significantly improved by ETN more than PLA; greatest effects on symptoms and feelings (ETN 60-62\% vs PLA 6\%; $P<0.0001$ ), and daily activities subscale (ETN $56-62 \%$ vs $1 \%$ PLA; $P<0.0001$ )

SF-36 at 12 weeks

- Mean physical component score was 52.7-52.8 for ETN vs 49.6 for PLA $(P<0.01)$

- Mean mental component score was 50.6-51.0 for ETN vs 46.5 for PLA $(P<0.01)$

- All 8 subscales were significantly improved by ETN more than PLA; greatest effects on bodily pain (ETN 6.2-7.1 vs PLA $1.1 ; P<0.0001$ ), and social function (ETN 3.9-6.0 vs PLA $0.5 ; P<0.0001)$

Improvements in DLQI at 12 weeks:

- $69.1 \%$ with ETN vs $22.1 \%$ with PLA $(P<0.0001)$

Depression assessed at 12 weeks:

- Mean difference in improvement in BDI between ETN and PLA was 1.8 (95\% Cl: 0.6, 2.9) $(P<0.0001)$

- Mean difference in improvement in Ham-D between ETN and PLA was $1.2(95 \% \mathrm{Cl}: 0.4,1.9)(P<0.0001)$

Fatigue assessed at 12 weeks:

- Mean difference in improvement in FACIT-F between ETN and PLA was $3.0(95 \% \mathrm{Cl}: 1.6,4.5)(P<0.0001)$

Mean improvement in DLQI: $68 \%$

Mean improvement in DLQI: $66 \%$

Mean DLQI score at start of OL (3.13) was maintained by switch to ETN $50 \mathrm{mg}$ (3.03)

aAbstract.

bSubanalysis of Singh et al. 2004 and van der Kerkhof et al. 2004a.

BDI, Beck Depression Inventory; biw, twice weekly; CI, confidence interval; DB, double-blind; DLQI, Dermatology Life Quality Index; ETN, etanercept; FACIT-F, functional assessment of chronic illness therapy-fatigue; Ham-D, Hamilton rating scale for depression; NS, nonsignificant; OL, open-label; OR, odds ratio; PC, placebo-controlled; PLA, placebo; PtGA, Patient's Global Assessment of psoriasis; R, randomized; SF-36, Short Form-36 Health Survey; qw, once weekly. 
Table 6 | Tolerability of etanercept in patients with psoriasis

\begin{tabular}{|c|c|c|c|c|}
\hline Level of evidence & Reference & Design & Treatment & Tolerability outcomes \\
\hline $1^{a}$ & Gottlieb et al. 2004b & $\begin{array}{l}\text { R, DB, PC, 12-week study } \\
\text { involving } 1347 \text { patients }\end{array}$ & $\begin{array}{l}\text { ETN } 25 \text { mg, } 50 \text { mg } \\
\text { biw, or PLA }\end{array}$ & $\begin{array}{l}\text { - ISR (low grade) was } 14 \% \text { for ETN vs } 6 \% \text { for PLA after } \\
12 \text { weeks. The rate in the ETN group was lower than } \\
\text { that seen in ETN studies in patients with RA ( } 37 \%) \\
\text { - } 1.8 \% \text { ETN- vs } 2 \% \text { of PLA-treated patients discontinued } \\
\text { treatment after AEs } \\
\text { - SAEs reported in } 1.7 \% \text { ETN- vs } 1.2 \% \text { in PLA-treated } \\
\text { patients } \\
\text { - Serious infections requiring hospitalization occurred } \\
\text { in } 0.3 \% \text { of ETN- vs } 1.2 \% \text { of PLA-treated patients }\end{array}$ \\
\hline 2 & Papp et al. 2005 & $\begin{array}{l}\text { R, DB, PC, } 24 \text {-week } \\
\text { study (OL phase during } \\
\text { weeks 13-24) involving } \\
583 \text { patients }\end{array}$ & $\begin{array}{l}\text { ETN } 25 \mathrm{mg} \text { or } 50 \mathrm{mg} \\
\text { biw, or PLA for } \\
12 \text { weeks. All patients } \\
\text { received ETN } 25 \mathrm{mg} \\
\text { biw during OL phase }\end{array}$ & $\begin{array}{l}\text { ISR (all mild or moderate) was the most commonly } \\
\text { reported event: } \\
\text { - } 16 \% \text { of ETN- vs } 6 \% \text { of PLA-treated patients after } \\
12 \text { weeks } \\
\text { - } 4 \% \text { of ETN- vs } 10 \% \text { of PLA-treated patients after } \\
24 \text { weeks }\end{array}$ \\
\hline
\end{tabular}

outside the USA where the endemic rate of the disease is greater (ACR 2003b). Simple screening procedures can be used to limit the risk of opportunistic infections, particularly tuberculosis, in new patients (ACR 2003b). Any patient who develops a new infection while undergoing treatment with etanercept should be monitored closely and treatment discontinued if the patient develops a serious infection or sepsis (Anon. 2006). There have been rare cases of new onset or exacerbations of existing demyelinating disorders following the use of TNF inhibitors. For example, 17 patients with rheumatoid arthritis developed neurologic events identified from the Adverse Events Reporting System FDA database. All these events were temporally related to TNF inhibitor therapy, but these resolved (partially or completely) after discontinuing the drug (Mohan et al. 2001). Therefore, prescribers should exercise caution when considering using etanercept in patients with preexisting or recent-onset central nervous system demyelinating disorders (Anon. 2006).

A meta analysis of three randomized controlled studies with etanercept in patients with psoriasis showed that no new or unanticipated patterns of adverse events occurred during the 12week treatment period (Gottlieb et al. 2004b, 2006). This analysis included 1347 patients with psoriasis, 1261 of whom received at least one dose of etanercept (25 mg once or twice weekly, or $50 \mathrm{mg}$ twice weekly) for a total of 933 patient-years of exposure. Discontinuation due to adverse events was similar in the pooled etanercept group (1.8\%) compared with placebo $(2.0 \%)$ after 12 weeks of treatment, and serious adverse events were experienced by similar proportions of patients in both groups ( $1.7 \%$ etanercept vs $1.2 \%$ placebo). Rates of serious infections requiring hospitalization were low $(0.3 \%$ etanercept vs $1.2 \%$ placebo) in both groups, and no opportunistic infections or cases of tuberculosis were reported. In addition, the safety profile of etanercept $50 \mathrm{mg}$ twice weekly in patients with psoriasis was comparable to that of patients treated with $25 \mathrm{mg}$ twice weekly (the approved regimen for patients with rheumatoid arthritis). Thus, over 12 weeks of treatment, the safety profile seen with etanercept in patients with psoriasis was comparable to that of placebo and was consistent with experiences in populations with rheumatoid arthritis (Gottlieb et al. 2004b, 2006).

\section{Economic evidence}

The economic evidence for the use of etanercept in psoriasis is limited to the models summarized in the NICE technology appraisal (NICE 2006), a paper from a US managed care perspective (Simpson et al. 2006), and an abstract (Nallamothu et al. 2004).

The NICE technology appraisal reports a model from the manufacturer of etanercept, and their own. The manufacturer's model was a Markov model using pooled data from three RCTs to evaluate the cost effectiveness of etanercept $25 \mathrm{mg}$ or $50 \mathrm{mg}$ twice weekly and topical therapy only. Quality-adjusted life-year (QALY) gain was estimated from improvements in DLQI between physician assessments over a 12-week period and eight further 12-week periods. The incremental cost-effectiveness ratio (ICER) for etanercept $25 \mathrm{mg}$ compared with topical therapy was $£ 125000$ per QALY over 12 weeks, and $£ 37200$ for intermittent therapy over 96 weeks. The ICER decreased to $£ 24000$ in patients with severe psoriasis and poor quality of life (PASI >10, DLQI >15). The NICE Technology Assessment Group model estimated QALY by mapping changes in DLQI to a nonspecific health-related quality of life instrument, EQ-5D. An intermittent regimen was assumed to be 3.2 12-week treatment cycles each year with 29-day intervals between cycles. The ICER for intermittent etanercept 25 mg was $£ 65320$ per QALY, decreasing to $£ 14460$ if the scenario included poor quality of life and hospitalization for nonresponders, defined as patients with PASI $\geq 10$ and DLQI $>10$ unresponsive to systemic therapy. Etanercept was more cost effective than efalizumab. Etanercept $50 \mathrm{mg}$ twice weekly was not considered to be cost effective. 
A US study investigated how changes to copayments for psoriasis treatments affect patients' expenses (Simpson et al. 2006). Out-of-pocket expenses were estimated to be $\$$ US1800 for phototherapy and \$US840 for etanercept, with corresponding costs to managed care organizations of \$US3008 and $\$$ US20300. Copayment structures are therefore favorable for patients receiving etanercept, but less so for managed care plans.

There is evidence from one meeting abstract that patients with moderate to severe psoriasis who have failed methotrexate therapy are likely to benefit from etanercept treatment in a reasonably cost-effective manner (Nallamothu et al. 2004). The study consisted of a computerized decision analytic model using a Markov process to assess benefits (QALY) and costs (\$US) for the treatment strategies of methotrexate alone or methotrexate followed by etanercept over 5 years. All cost estimates were obtained from one center. The methotrexate strategy treatment consisted of a maintenance dose of $15 \mathrm{mg} /$ week if $20 \mathrm{mg} /$ week was successful during a 3-month induction phase. If the induction phase was unsuccessful, then patients were started on etanercept $25 \mathrm{mg}$ twice weekly for 3 months and continued on a maintenance regimen of $25 \mathrm{mg}$ weekly. However, as the recommended approved maintenance dose is $50 \mathrm{mg}$ weekly, the cost of this treatment strategy may be underestimated (Anon. 2006).

The analysis showed that methotrexate alone costs approximately \$US17 400 for a net benefit of 2.8 QALYs. In comparison, the methotrexate/etanercept strategy was $44 \%$ more costly (\$US25 000) but resulted in a greater benefit (3.1 QALYs). Thus, the ICER for the methotrexate/etanercept strategy was \$US25 000 per QALY (i.e. a cost difference of $\$$ US7600 for a net benefit of 0.3 QALY). This value is sensitive to estimates of the long-term efficacy and cost of etanercept (Nallamothu et al. 2004). Therefore, although improved benefit may be achieved with the methotrexate/etanercept strategy, there are additional cost implications which may have to be evaluated on a case-by-case basis.

\section{Resource utilization}

Because of shortcomings of some of the standard established treatments for psoriasis, the demand for newer, more effective, more convenient and better-tolerated treatments is likely to be high (Smith et al. 2005). The acquisition cost of etanercept of more than \$US12 000 per year is much higher than for other oral systemic treatments for psoriasis (Lebwohl 2003). In contrast, the average individual cost of phototherapy has recently been estimated as $€ 325.00$ (range $€ 57.20-972.40$ ) (Langan et al. 2004). Thus, it will be important to establish a rationale for developing a long-term cost model to assess the use and associated economics for the use of etanercept (and other targeted biologics) for the treatment of psoriasis. Considerations for evaluating the costs of these agents should include the ability to maintain off-treatment remissions or clearing, improvements in patients' quality of life, and better safety and tolerability profiles (Rich 2004).

At present comprehensive economic data on etanercept in psoriasis are limited, and its long-term effect on the burden of the disease is unknown. It will be important to determine in future studies the drug's effect on reductions in direct and indirect costs of the disease. For example, successful treatment of severe disease may result in significant reductions in visits to physicians, length of hospital stay in some cases, and work days lost to the disease or treatments. Until studies assess these resources, costs, and outcomes, the overall effect of etanercept will be difficult to determine. Whether the benefits gained from etanercept justify its acquisition cost will be a matter for decision makers in individual healthcare systems.

\section{Patient group/population}

In the USA, etanercept is licensed for the treatment of adult patients with chronic moderate to severe plaque psoriasis who are candidates for systemic therapy or phototherapy. In contrast, UK guidelines indicate that patients should have severe disease and have either failed or be intolerant to established therapies to be considered eligible for treatment (Smith et al. 2005). Patients treated with etanercept should be withdrawn from therapy after 12 weeks if there has not been at least a 50\% improvement in PASI (or body surface area involvement) score from baseline (Smith et al. 2005). NICE recommends the use of etanercept at a dose not exceeding $25 \mathrm{mg}$ twice weekly in patients with a PASI $\geq 10$ and DLQI $>10$, whose psoriasis has failed to respond to, is intolerant to, or has a contraindication to, systemic therapies (NICE 2006). Again, treatment should be discontinued after 12 weeks if there has not been a $\geq 75 \%$ improvement in PASI, or a $\geq 50 \%$ improvement in PASI plus a 5-point reduction in DLQI. A European expert panel recently recommended initiating treatment with $50 \mathrm{mg}$ twice weekly, and maintaining until remission for a maximum of 24 weeks; retreatment is dependent on the physician's assessment (Boehncke et al. 2006).

At present there is limited (level 5 only) evidence from case studies that etanercept is effective in some other types of psoriasis. Etanercept $25 \mathrm{mg}$ twice weekly was effective in the treatment of pustular psoriasis (Kamarashev et al. 2002) and recalcitrant palmoplantar psoriasis unresponsive to other treatments, including topical therapy and phototherapy (Weinberg 2003).

\section{Dosage, administration, and formulations}

In the USA, etanercept is indicated for the treatment of adult patients (18 years or older) with chronic moderate to severe plaque psoriasis who are candidates for systemic therapy or phototherapy. It is supplied in a single-use prefilled $1 \mathrm{~mL}$ syringe as a sterile, preservative-free solution containing $50 \mathrm{mg} / \mathrm{mL}$ etanercept, or as multiple-use $1 \mathrm{~mL}$ vial of etanercept $25 \mathrm{mg} / \mathrm{mL}$ for subcutaneous injection.

In the US, the recommended starting dose of etanercept for adult patients is $50 \mathrm{mg}$ twice weekly (administered 3 or 4 days apart) for 3 months, followed by a reduction to a maintenance dose of $50 \mathrm{mg}$ per week. In the EU, the recommended is $25 \mathrm{mg}$ twice weekly, or $50 \mathrm{mg}$ twice weekly for up to 12 weeks followed if necessary by $25 \mathrm{mg}$ twice weekly. Treatment should continue until remission for up to 24 weeks, and should be discontinued if no response has been achieved within 12 weeks. 
Patients are encouraged to self-administer etanercept. The most common locations for subcutaneous injections are the abdomen, middle thigh, and the outer area of the upper arms. So that injection site reactions are minimized, patients are instructed to rotate the site for each injection. Areas where the skin is tender, bruised, red, or hard or where there are scars or stretch marks should be avoided (Anon. 2006).

\section{Clinical value}

There is good evidence from a number of large, good quality clinical studies showing that treatment with etanercept for up to 24 weeks improved physician-assessed clinical outcomes when compared with placebo. These outcomes were seen as soon as two to four weeks from initiating treatment. More patients treated with etanercept had improvements in their disease score (PASI) and achieved clearance compared with placebo. On stopping treatment in clinical studies the mean time to relapse was 3 months, with a quarter of patients not experiencing relapse until at least after 5 months.

One of the most debilitating aspects of severe psoriasis is the effect that it has on patients' quality of life and mental and physical function. There is good evidence that significant improvements in these patient-oriented outcomes have been achieved with etanercept in clinical studies. Improvements in scores of both disease-specific (DLQI) and generic (SF-36) instruments have been achieved. Limited evidence has also emerged recently suggesting that etanercept can improve symptoms associated with depression and fatigue, which are often experienced in patients with psoriasis.

There is limited evidence that etanercept can be used in combination therapy. Although the combination of etanercept and methotrexate has been used successfully for the treatment of rheumatoid arthritis and psoriatic arthritis, phase III and IV studies in patients with psoriasis with this and other combinations are still underway.

Experience with TNF inhibitors has raised some clinical concern regarding risks of opportunistic infections and malignancy. Most of the evidence related to the safety and tolerability of etanercept has been gained from its long-term use in patients with rheumatoid arthritis. In contrast, many fewer patients with psoriasis have been exposed to the drug. However, the evidence so far suggests that the safety profile in both patient populations is similar. The most commonly experienced adverse event is an injection site reaction which may occur in up to $20 \%$ of patients. Typically such a reaction occurs within the first month of treatment and spontaneously resolves. Experience with patients with rheumatoid arthritis has shown that serious adverse effects may be avoided or limited by following suitable screening procedures and published guidelines when considering using etanercept. Nevertheless, long-term monitoring will be required to determine the potential for increased risk of infection and malignancy in of TNF inhibitor-treated patients with psoriasis.

In conclusion, patients with moderate to severe psoriasis who are candidates for systemic therapy or phototherapy now have the option of effective and convenient treatment with etanercept. Because of its higher acquisition cost compared with existing systemic treatments, more economic studies and modeling will be required to define its cost effectiveness in the disease.

\section{References}

ACR. FDA meeting March 2003: update on the safety of new drugs for rheumatoid arthritis. Hotline. March 2003a. Available at: http://www.rheumatology.org/publications/hotline/0303TNFL.asp?aud=mem (accessed November 30, 2005).

ACR. FDA meeting March 2003: update on the safety of new drugs for rheumatoid arthritis. Part II: CHF, infection and other safety issues. March 2003b. Hotline. Available at:

http://www.rheumatology.org/publications/hotline/0803chf.asp?aud=mem (accessed November 30, 2005).

Anon. Enbrel US Prescribing Information. Available at http://www.enbrel.com/prescribing-information.jsp (accessed October 6, 2006).

Ashcroft DM, Wan Po AL, Williams HC, Griffiths CE. Clinical measures of disease severity and outcome in psoriasis: a critical appraisal of their quality. Br J Dermatol. 1999;141:185-191.

Boehncke WH, Brasie RA, Chimenti S, et al ; European Dermatology Expert Group. Recommendations for the use of etanercept in psoriasis: a European dermatology expert group consensus. J Eur Acad Dermatol Venereol. 2006;20:988-998

Bonifati C, Ameglio F. Cytokines in psoriasis. Int J Dermatol. 1999;38:241-251. Cassano N, Loconsole F, Galluccio A, Miracapillo A, Pezza M, Vena GA. Once-weekly administration of high-dosage etanercept in patients with plaque psoriasis: results of a pilot experience (power study). Int J Immunopathol Pharmacol. 2006;19:225-229.

Elewski B, Leonardi C, van der Kerkhof $\mathrm{P}$, et al. Clinical and pharmacokinetic equivalence of etanercept $25 \mathrm{mg}$ twice weekly and $50 \mathrm{mg}$ once weekly in patients with psoriasis. J Invest Dermatol. 2005;125:A2. Abstract 911.

Finlay AY. Current severe psoriasis and the rule of tens. $\mathrm{Br} J$ Dermatol. 2005;152:861-867.

Gordon K, Norman N, Frankel E, et al. Efficacy of etanercept in an integrated multistudy databse of patients with psoriasis. J Invest Dermatol. 2004;122:A54. Abstract 321.

Gordon KB, Gottlieb AB, Leonardi CL, et al. Clinical response in psoriasis patients discontinued from and then reinitiated on etanercept therapy. J Dermatolog Treat. 2006;17:9-17.

Gottlieb AB, Matheson RT, Lowe N, et al. A randomized trial of etanercept as monotherapy for psoriasis. Arch Dermatol. 2003;139:1627-1632.

Gottlieb AB, Gordon K, Gaspari AA, et al. Clinical and pathologic improvements in patients with psoriasis following etanercept monotherapy. J Invest Dermatol. 2004a;123:A65. Abstract 388 .

Gottlieb AB, Goffe B, Veith J, et al. Safety of etanercept in an integrated multistudy database of patients with psoriasis. J Invest Dermatol. 2004b;122:A55. Abstract 325.

Gottlieb A, Gordon K, Wang A, et al. Durability of treatment response following withdrawal from etanercept in psoriasis patients. J Invest Dermatol. 2004c;122:A51. Abstract 306.

Gottlieb AB, Leonardi CL, Goffe BS, et al. Etanercept monotherapy in patients with psoriasis: a summary of safety, based on an integrated multistudy database. J Am Acad Dermatol. 2006;54(Suppl. 2):S92-S100.

Hochberg MC, Lebwohl MG, Plevy SE, Hobbs KG, Yocum DE. The benefit/risk profile of TNF-blocking agents: findings of a consensus panel. Semin Arthritis Rheum. 2005;34:819-836.

Jacob SE, Sergay A, Kerdel FA. Etanercept and psoriasis, from clinical studies to real life. Int J Dermatol. 2005;44:688-691.

Javitz HS, Ward MM, Farber E, Nail L, Vallow SG. The direct cost of care for psoriasis and psoriatic arthritis in the United States. $\mathrm{J}$ Am Acad Dermatol. 2002;46:850-860 
Kamarashev J, Lor P, Forster A, Heinzerling L, Burg G, Nestle FO. Generalised pustular psoriasis induced by cyclosporin $A$ withdrawal responding to the tumour necrosis factor alpha inhibitor etanercept. Dermatology. 2002;205:213-216.

Krueger G, Koo J, Lebwohl M, Menter A, Stern RS, Rolstad T. The impact of psoriasis on quality of life: results of a 1998 National Psoriasis Foundation patient-membership survey. Arch Dermatol. 2001;137:280-284.

Krueger GG, Langley RG, Finlay AY, et al. Patient-reported outcomes of psoriasis improvement with etanercept therapy: results of a randomized phase III trial. Br J Dermatol. 2005;153:1192-1199.

Krueger GG, Elewski B, Papp K, Wang A, Zitnik R, Jahreis A. Patients with psoriasis respond to continuous open-label etanercept treatment after initial incomplete response in a randomized, placebo-controlled trial. J Am Acad Dermatol. 2006;54(Suppl. 2):S112-S119.

Langan SM, Heerey A, Barry M, Barnes L. Cost analysis of narrowband UVB phototherapy in psoriasis. J Am Acad Dermatol. 2004;50:623-626.

Langley RG, Ellis CN. Evaluating psoriasis with Psoriasis Area and Severity Index, Psoriasis Global Assessment, and Lattice Systems Physician's Global Assessment. J Am Acad Dermatol. 2004;51:563-569.

Lebwohl M. Psoriasis. Lancet. 2003;361:1197-1204.

Leonardi CL, Powers JL, Matheson RT, et al; Etanercept Psoriasis Study Group. Etanercept as monotherapy in patients with psoriasis. $N$ Engl $\mathrm{J} \mathrm{Med.}$ 2003;349:2014-2022.

Leonardi CL, Elewski B, Wang A, et al. The efficacy and safety of etanercept in the rettreatment of psoriasis after relapse. J Invest Dermatol. 2004;122:A52. Abstract 312.

Mease PJ, Goffe BS, Metz J, Van der Stoep A, Finck B, Burge DJ. Etanercept in the treatment of psoriatic arthritis and psoriasis: a randomised trial. Lancet. 2000;356:385-390.

Mohan N, Edwards ET, Cupps TR, et al. Demyelination occurring during anti-tumor necrosis factor alpha therapy for inflammatory arthritides. Arthritis Rheum. 2001;44:2862-2869.

Nallamothu PG, Nallamothu BK, Lim HW. Etanercept in patients with moderate to severe psoriasis who fail methotrexate: a cost-effectiveness analysis. J Invest Dermatol. 2004;122:A63. Abstract 378.

NICE (National Institute for Health and Clinical Excellence). Technology Appraisal Guidance 103. Etanercept and efalizumab for the treatment of adults with psoriasis. London: National Institute for Health and Clinical Excellence; July 2006. Available at: http://www.nice.org.uk/page.aspx?o=TA103 (accessed July 29, 2006).

Nayak AS, Zitnik R. enbrel (etanercept) monotherapy in patients with psoriasis: clinical and pathologic improvements. J Allergy Clin Immunol. 2003;111:S273. Abstract 819.

Papp KA, Tyring S, Lahfa M, et al; Etanercept Psoriasis Study Group. A global phase III randomized controlled trial of etanercept in psoriasis: safety, efficacy, and effect of dose reduction. Br J Dermatol. 2005;152:1304-1312.

Pardasani AG, Feldman SR, Clark AR. Treatment of psoriasis: an algorithmbased approach for primary care physicians. Am Fam Physician. 2000;61:725-733.
Rapp SR, Feldman SR, Exum ML, Fleischer AB Jr, Reboussin DM. Psoriasis causes as much disability as other major medical diseases. $J$ Am Acad Dermatol. 1999;41:401-407.

Rich SJ. Considerations for assessing the cost of biologic agents in the treatment of psoriasis. J Manag Care Pharm. 2004;10(Suppl. B):S38-S41.

Shikiar R, Bresnahan BW, Stone SP, Thompson C, Koo J, Revicki DA. Validity and reliability of patient reported outcomes used in psoriasis: results from two randomized clinical trials. Health Qual Life Outcomes. 2003;1:53.

Simpson JF, Yelverton CB, Balkrishnan R, Fleischer A Jr, Lide W, Feldman SR. Would elimination of copayments for phototherapy decrease the cost of treating psoriasis with systemic biologics? A cost analysis. Manag Care Interface. 2006;19:39-43.

Singh A, Ganguly R, Sato R. Clinically meaningful improvement in dermatology life quality index in patients with chronic plaque psoriasis: a meta-analytic summary of randomized clinical trials of etanercept. $J$ Invest Dermatol. 2004;123:A69. Abstract 409.

Smith $\mathrm{CH}$, Anstey AV, Barker JN, et al; British Association of Dermatologists. British Association of Dermatologists guidelines for use of biological interventions in psoriasis 2005. Br J Dermatol. 2005;153:486-497.

Stern RS. Utilization of outpatient care for psoriasis. J Am Acad Dermatol. 1996;35:543-545.

Stern RS, Nijsten T, Feldman SR, Margolis DJ, Rolstad T. Psoriasis is common, carries a substantial burden even when not extensive, and is associated with widespread treatment dissatisfaction. $J$ Investig Dermatol Symp Proc. 2004;9:136-139.

Strober B, Yamauchi P, Korman N, et al. The efficacy of etanercept in psoriasis patients with varying treatment histories. J Invest Dermatol. 2004;122:A43. Abstract 254.

Tyring S, Gottlieb A, Papp K, et al. Etanercept and clinical outcomes, fatigue, and depression in psoriasis: double-blind placebo-controlled randomized phase III trial. Lancet. 2006;367:29-35.

van der Kerkhof PCM, Sterry W, Ortonne J-P, et al. Etanercept efficacy results from an integrated multistudy in patients with psoriasis. $J$ Invest Dermatol. 2004a;123:A65. Abstract 389.

van der Kerkhof PCM, Sterry W, Ortonne J-P, et al. Etanercept improves quality of life in patients with chronic plaque psoriasis: a meta-analytic summary of randomized clinical trials with etanercept. J Invest Dermatol. 2004b;123:A69. Abstract 408.

Weinberg JM. Successful treatment of recalcitrant palmoplantar psoriasis with etanercept. Cutis. 2003;72:396-398.

Correspondence: Paul Chrisp, Core Medical Publishing, Mere House, Brook Street, Knutsford, Cheshire WA16 8GP, UK or at editor@coreevidence.com 